Supplement of Biogeosciences, 15, 2055-2073, 2018

https://doi.org/10.5194/bg-15-2055-2018-supplement

(C) Author(s) 2018. This work is distributed under

the Creative Commons Attribution 4.0 License.

(c) (1)

Supplement of

\title{
Variability in copepod trophic levels and feeding selectivity based on stable isotope analysis in Gwangyang Bay of the southern coast of the Korean Peninsula
}

Mianrun Chen et al.

Correspondence to: Chang-Keun Kang (ckkang@gist.ac.kr)

The copyright of individual parts of the supplement might differ from the CC BY 4.0 License. 
Table S1: Body length, living environment and the empirical equation between dry weight $(D W)$ and body length $(L)$.

\begin{tabular}{|c|c|c|c|c|}
\hline Species & $L(\mathrm{~mm})$ & Environment & Equation & Reference \\
\hline Acartia hudsonica & $0.67-1.32$ & Marine & $\ln D W=3.09 \ln L-19.19$ & Chisholm and Roff (1990); Walter (2010a) \\
\hline Acartia omorii & $0.81-1.22$ & Marine & $\ln D W=3.09 \ln L-19.19$ & Chisholm and Roff (1990); Copepoda (2008a) \\
\hline Acartia ohtsukai & $1.03-1.23$ & Marine, & $\ln D W=3.09 \ln L-19.19$ & Chisholm and Roff (1990); Walter (2014a) \\
\hline Acartia erythraea & $1.15-1.25$ & $\begin{array}{l}\text { Brackish } \\
\text { Marine, } \\
\text { Brackish }\end{array}$ & $\ln D W=3.09 \ln L-19.19$ & Chisholm and Roff (1990); Copepoda (2014a) \\
\hline Bestiolina coreana & $0.85-0.96$ & Marine & $\ln W=3.25 \ln L-19.65$ & Chisholm and Roff (1990); Walter (2010b) \\
\hline Calanus sinicus & $2.07-3.6$ & Marine & $\log D W=2.66 \log L-6.68$ & Copepoda (2008b); Uye (1982) \\
\hline Clausocalanus furcatus & $0.7-1.31$ & Marine & $\ln W=3.25 \ln L-19.65$ & Boxshall (2004); Chisholm and Roff (1990) \\
\hline Centropages abdominalis & $1.43-1.78$ & Marine & $\log D W=3.00 \log L-8.08$ & Copepoda (2008c); Uye (1982) \\
\hline Centropages dorsispinatus & $1.25-1.4$ & Marine & $\log D W=3.00 \log L-8.08$ & Copepoda (2008d); Uye (1982) \\
\hline Paraeuchaeta plana & $2.84-3.30$ & Marine & $\log D W=2.66 \log L-6.68$ & Boxshall and Walter (2014); Uye (1982) \\
\hline Eurytemora Pacifica & $0.92-1.82$ & $\begin{array}{l}\text { Marine, } \\
\text { Brackish }\end{array}$ & $D W=1.471 * 10^{-8} L^{3.064}$ & $\begin{array}{l}\text { Walter (2014b), Equation Follows Temora } \\
\text { turbinata (Ara, 2001) }\end{array}$ \\
\hline Paracalanus parvus & $0.60-1.30$ & $\begin{array}{l}\text { Marine, } \\
\text { Brackish }\end{array}$ & $\ln D W=3.25 \ln L-19.65$ & $\begin{array}{l}\text { Chisholm and Roff (1990); Walter and Boxshall } \\
\text { (2014) }\end{array}$ \\
\hline Paracalanus aculeatus & $0.78-1.36$ & Marine & $\ln D W=3.25 \ln L-19.65$ & Boxshall (2008); Chisholm and Roff (1990) \\
\hline Pseudodiaptomus koreanus & $1.09-1.45$ & $\begin{array}{l}\text { Marine, } \\
\text { Brackish }\end{array}$ & $D W=1.306 * 10^{-9} L^{3.361}$ & Ara (2001); Soh et al. (2012); Walter (2014c) \\
\hline Pseudodiaptomus marinus & $0.95-1.58$ & $\begin{array}{l}\text { Marine, } \\
\text { Brackish }\end{array}$ & $D W=1.306 * 10^{-9} L^{3.361}$ & Ara (2001); Walter (2014d) \\
\hline Sinocalanus tenellus & $1.30-1.45$ & $\begin{array}{l}\text { Marine, } \\
\text { Brackish }\end{array}$ & $\log D W=3.00 \log L-7.42$ & Walter (2013a); Uye (1982) \\
\hline Labidocera rotunda & $1.58-2.31$ & Marine & $D W=3.770 * 10^{-8} L^{2.637}$ & Ara (2001); Walter (2008a) \\
\hline Labidocera euchaeta & $2.41-2.79$ & Marine & $D W=3.770 * 10^{-8} L^{2.637}$ & Ara (2001); Copepoda (2008e) \\
\hline Tortanus dextrilobatus & $1.53-2.05$ & Marine & $\log D W=2.50 \log L-6.11$ & Uye (1982); Walter (2013b) \\
\hline Tortanus forcipatus & $1.20-1.27$ & Marine & $\log D W=2.50 \log L-6.11$ & Uye (1982); Walter (2013c) \\
\hline Corycaeus affinis & $0.62-0.87$ & Marine & $\ln D W=1.7 \ln L-9.92$ & Chisholm and Roff (1990); Copepoda (2014b) \\
\hline Oithona davisae & $0.47-0.61$ & $\begin{array}{l}\text { Marine, } \\
\text { Brackish, } \\
\text { Fresh }\end{array}$ & $\ln D W=1.10 \ln L-7.07$ & $\begin{array}{l}\text { Chisholm and Roff (1990); Ferrari and Orsi } \\
\text { (1984); Walter (2008b) }\end{array}$ \\
\hline Oncaea venella & $0.55-1.23$ & Marine & $\ln D W=1.96 \ln L-11.64$ & $\begin{array}{l}\text { Chisholm and Roff (1990); Walter and Böttger- } \\
\text { Schnack (2011) }\end{array}$ \\
\hline
\end{tabular}

\section{References}

Ara, K.: Length-weight relationships and chemical content of the planktonic copepods in the Cananeia Lagoon estuarine 5 system, São Paulo, Brazil. Plankton Bio. Ecol., 48,121-127, 2001. 
Boxshall, G.: Clausocalanus furcatus (Brady, 1883), in: World of Copepods database, Walter, T.C. and Boxshall, G. 2017, available at http://www.marinespecies.org/copepoda, 2004.

Boxshall, G.: Paracalanus aculeatus Giesbrecht, 1888. In: World of Copepods database. Walter, T.C. and Boxshall, G., 2017 available at http://www.marinespecies.org/copepoda, 2008.

5 Boxshall, G. and Walter, T. C.: Paraeuchaeta plana (Mori, 1937), in: World of Copepods database, Walter, T.C. and Boxshall, G., 2017, available at http://www.marinespecies.org/copepoda/, 2014.

Chisholm, L.A. and Roff, J.C.: Size-weight relationships and biomass of tropical neritic copepods off Kingston, Jamaica. Mar. Biol., 106, 71-77, 1990.

Copepoda: Acartia (Acartiura) omorii Bradford, 1976, in: World of Copepods database, Walter, T.C. and Boxshall, G., 2017,

10 available at http://www.marinespecies.org/copepoda/, 2008a.

Copepoda: Calanus sinicus Brodsky, 1962, in: World of Copepods database, Walter, T.C. and Boxshall, G., 2017, available at http://www.marinespecies.org/copepoda/, 2008b.

Copepoda: Centropages abdominalis Sato, 1913, in: World of Copepods database Walter, T.C. and Boxshall, G., 2017, aavailable at http://www.marinespecies.org/copepoda/, 2008c.

15 Copepoda: Centropages dorsispinatus Thompson I.C. \& Scott A., 1903, in: World of Copepods database, Walter, T.C. and Boxshall, G., 2017, Available at http://www.marinespecies.org/copepoda/, 2008d.

Copepoda: Labidocera euchaeta Giesbrecht, 1889, in: World of Copepods database, Walter, T.C. and Boxshall, G., 2017, available at http://www.marinespecies.org/copepoda/, 2008e.

Copepoda: Acartia (Odontacartia) erythraea Giesbrecht, 1889, in: World of Copepods database. Walter, T.C. and Boxshall,

20 G., 2017, available at http://www.marinespecies.org/copepoda/, 2014a.

Copepoda: Corycaeus affinis McMurrich, 1916. In: World of Copepods database, Walter, T.C. and Boxshall, G., 2017, available at http://www.marinespecies.org/copepoda/, 2014b.

Ferrari, F.D. and Orsi., J.J.: Oithona davisae, new species, and Limnoithona sinensis (Burckhardt, 1912) (Copepoda: Oithonidae) from the Sacramento-San Joaquin Estuary, California. J. Crustacean Biol. 4, 106-126, 1984.

25 Soh, H.Y., Kwon, S.W., Lee, W., and Yoon, Y.H.: A new Pseudodiaptomus (Copepoda, Calanoida) from Korea supported by molecular data. Zootaxa, 3368, 229-244, 2012.

Uye, S.: Length-weight relationships of important zooplankton from the inland sea of Japan. J. Oceanogr. Soc. Japan, 38, 149$158,1982$.

Walter, T.C.: Labidocera rotunda Mori, 1929, in: World of Copepods database, Walter, T.C. and Boxshall, G., 2017, available 30 at http://www.marinespecies.org/copepoda/, 2008a.

Walter, T.C: Oithona davisae Ferrari F.D. \& Orsi, 1984, in: World of Copepods database, Walter, T.C. and Boxshall, G., 2017, available at http://www.marinespecies.org/copepoda/, 2008b.

Walter, T.C: Acartia hudsonica Pinhey, 1926, in: World of Copepods database, Walter, T.C. and Boxshall, G., 2017, available at http://www.marinespecies.org/copepoda/, 2010a. 
Walter, T.C: Bestiolina coreana Moon, Lee \& Soh, 2010. in: World of Copepods database, Walter, T.C. and Boxshall, G., 2017, available at http://www.marinespecies.org/copepoda/, $2010 \mathrm{~b}$.

Walter, T.C. and Böttger-Schnack, R.: Oncaea venus tavenella Farran, 1929, in: World of Copepods database, Walter, T.C. and Boxshall, G., 2017, available at http://www.marinespecies.org/copepoda/, 2011.

5 Walter, T.C.: Sinocalanus tenellus (Kikuchi K., 1928), in: World of Copepods database, Walter, T.C. and Boxshall, G., 2017, available at http://www.marinespecies.org/copepoda/, 2013a.

Walter, T.C.: Tortanus (Eutortanus) dextrilobatus Chen \& Zhang, 1965. in: World of Copepods database, Walter, T.C. and Boxshall, G., 2017, available at http://www.marinespecies.org/copepoda/, $2013 \mathrm{~b}$.

Walter, T.C.: Tortanus (Tortanus) forcipatus (Giesbrecht, 1889), in: World of Copepods database, Walter, T.C. and Boxshall,

10 G., 2017, available at http://www.marinespecies.org/copepoda/, 2013c.

Walter, T.C.: Acartia (Odontacartia) ohtsukai Ueda \& Bucklin, 2006, in: World of Copepods database, Walter, T.C. and Boxshall, G., 2017, available at http://www.marinespecies.org/copepoda/, 2014a.

Walter, T.C.: Eurytemora pacifica Sato, 1913, in: World of Copepods database, Walter, T.C. and Boxshall, G., 2017, available at http://www.marinespecies.org/copepoda/, 2014b.

15 Walter, T.C.: Pseudodiaptomus koreanus Soh, Kwon, Lee \& Yoon, 2012, in: World of Copepods database, Walter, T.C. and Boxshall, G., 2017, available at http://www.marinespecies.org/copepoda/, 2014c.

Walter, T.C.: Pseudodiaptomus marinus Sato, 1913, in: World of Copepods database, Walter, T.C. and Boxshall, G., 2017, available at http://www.marinespecies.org/copepoda/, 2014d.

Walter, T.C. and Boxshall, G.: Paracalanus parvus (Claus, 1863), in: World of Copepods database, Walter, T.C. and Boxshall, 20 G., 2017, available at http://www.marinespecies.org/copepoda/, 2014. 
Table S2: Abundance composition of copepods in Gwangyang Bay in 2015 (ind. $\mathbf{m}^{-3}$ ).

\begin{tabular}{|c|c|c|c|c|c|c|c|c|c|}
\hline \multirow[t]{2}{*}{ Species/Seasons } & \multicolumn{9}{|c|}{ Stations } \\
\hline & $\# 1$ & $\# 2$ & $\# \mathbf{3}$ & $\# 4$ & $\# 5$ & $\# 6$ & $\# 7$ & $\# \mathbf{8}$ & $\# 9$ \\
\hline \multicolumn{10}{|l|}{ Winter } \\
\hline Acartiahudsonica & 29.5 & 489.2 & 188.7 & & & & & & \\
\hline Acartiaomorii & & & 310.8 & 735.4 & 1413.9 & 687.5 & 400.0 & 566.2 & 522.0 \\
\hline Calanussinicus & & & 5.6 & & 35.9 & 27.0 & 39.4 & 46.0 & 120.5 \\
\hline Centropages abdominalis & & & 66.6 & 227.8 & 275.6 & 546.0 & 259.1 & 497.1 & 650.5 \\
\hline Eurytemora pacifica & 5.9 & 17.4 & 44.4 & & & & & & \\
\hline Paracalanus parvus & 8.8 & 19.9 & 194.3 & 370.9 & 730.9 & 498.8 & 692.9 & 639.8 & 939.6 \\
\hline Pseudodiaptomus koreanus & & 2.5 & & & & & & & \\
\hline Sinocalanus tenellus & 8.8 & & & & & & & & \\
\hline Corycaeus affinis & & & 5.6 & 52.1 & 83.9 & 128.1 & 73.2 & 41.4 & 64.2 \\
\hline Oithona davisae & & 9.9 & 33.3 & 91.1 & 191.7 & 128.1 & 123.9 & 244.0 & 337.3 \\
\hline Nauplii & & & & 26.0 & 95.9 & 67.4 & 67.6 & 124.3 & 48.2 \\
\hline Unid. harpacticods & 11.8 & 12.4 & 94.4 & 110.6 & 59.9 & 40.4 & 16.9 & 0.0 & 8.0 \\
\hline \multicolumn{10}{|l|}{ Spring } \\
\hline Acartia hudsonica & & & 169.4 & 57.2 & 12.9 & 24.9 & 17.4 & 29.4 & 114.8 \\
\hline Acartia ohtsukai & & & 29.0 & 5.7 & & & & & \\
\hline Calanus sinicus & & & 33.9 & 31.5 & 81.5 & 145.2 & 14.5 & 44.1 & 3.1 \\
\hline Paracalanus parvus & & 5.0 & 29.0 & 37.2 & 10.7 & 12.4 & 2.9 & 2.5 & 4.7 \\
\hline Pseudodiaptomus koreanus & 1981.6 & 79.5 & 33.9 & 65.8 & 10.7 & & & & \\
\hline Pseudodiaptomus marinus & & & & & & 16.6 & & & \\
\hline Sinocalanus tenellus & 283.1 & 9.9 & 7.3 & 2.9 & & & & & \\
\hline Labidocera rotunda & & & & & 4.3 & 8.3 & & & 1.6 \\
\hline Tortanus dextrilobatus & & 79.5 & 4.8 & & & & & & \\
\hline Tortanus forcipatus & & & 2.4 & & 4.3 & & 2.9 & 2.5 & \\
\hline Corycaeus affinis & & & 9.7 & 123.0 & 137.3 & 145.2 & 104.5 & 53.9 & 15.7 \\
\hline Oithona davisae & & & & & & & & & 1.6 \\
\hline Nauplii & & & 2.4 & & & & & & \\
\hline \multicolumn{10}{|l|}{ Summer } \\
\hline Acartia ohtsukai & 11.3 & 1326.5 & 553.7 & 871.8 & 133.3 & & & & \\
\hline Acartia erythraea & & & & 37.9 & 42.8 & 82.8 & 26.7 & 109.3 & 39.8 \\
\hline Calanus sinicus & & & & 14.2 & 7.5 & 1.3 & 1.4 & & \\
\hline Paracalanus parvus & & 16.2 & & 19.0 & 12.6 & 6.7 & & & \\
\hline Sinocalanus tenellus & & 16.2 & & & & & & & \\
\hline Labidocera rotunda & & & & & & & 43.6 & 6.2 & 32.7 \\
\hline Labidocera euchaeta & & & 1.8 & 61.6 & 22.6 & 26.7 & & & \\
\hline
\end{tabular}




\begin{tabular}{|c|c|c|c|c|c|c|c|c|c|}
\hline Tortanus dextrilobatus & 41.5 & 1844.1 & 226.9 & 364.8 & 125.7 & & & & \\
\hline Tortanus forcipatus & & & & 28.4 & 17.6 & 4.0 & & 1.6 & \\
\hline Corycaeus affinis & & & & & & 1.3 & & & \\
\hline Oithona davisae & & & & & & 1.3 & & & \\
\hline Unid. harpacticods & & & & & & 1.4 & & & \\
\hline \multicolumn{10}{|l|}{ Autumn } \\
\hline Acartia omorii & & 3.1 & 4.1 & 1.7 & 0.9 & 4.1 & 4.5 & 0.4 & 0.9 \\
\hline Acartia ohtsukai & & 2.3 & 3.3 & & & & 1.0 & 0.8 & 0.9 \\
\hline Acartia erythraea & & 0.9 & 4.9 & & 0.9 & 1.2 & & & 0.3 \\
\hline Calanus sinicus & & & & & 0.5 & 5.4 & 13.1 & 20.1 & 7.0 \\
\hline Centropages abdominalis & & 0.0 & 0.8 & & & 0.4 & & & \\
\hline Centropages dorsispinatus & & 2.4 & 0.8 & & & 0.4 & 1.0 & 0.8 & \\
\hline Paracalanus parvus & & 7.1 & 10.6 & 6.1 & 1.4 & 2.1 & 10.0 & 9.4 & 1.8 \\
\hline Paracalanus aculeatus & & & & & & & 0.5 & & 0.3 \\
\hline Pseudodiaptomus koreanus & 3.6 & & & & & & & & \\
\hline Pseudodiaptoтиs тагіпиs & & & & & & & & & 0.9 \\
\hline Sinocalanus tenellus & 13.3 & & & & & & & & \\
\hline Labidocera rotunda & & & & & & 0.8 & & & 0.3 \\
\hline Tortanus forcipatus & & & & 2.6 & 2.8 & 4.1 & 0.5 & 0.4 & \\
\hline Paraeuchaeta plana & & & & & & & 1.5 & 2.0 & 1.8 \\
\hline Corycaeus affinis & & 3.6 & 5.7 & 21.0 & 11.1 & 22.3 & 80.3 & 83.0 & 7.0 \\
\hline Oithona davisae & & & & & 0.5 & & & 0.4 & 0.9 \\
\hline Oncaea venella & & & & & & & 0.5 & 0.4 & 0.3 \\
\hline Bestiolina coreana & & & 3.3 & & & & & & \\
\hline Nauplii & & & & & & & & & 0.6 \\
\hline Unid. harpacticods & & 2.6 & & 0.9 & & & & & \\
\hline
\end{tabular}



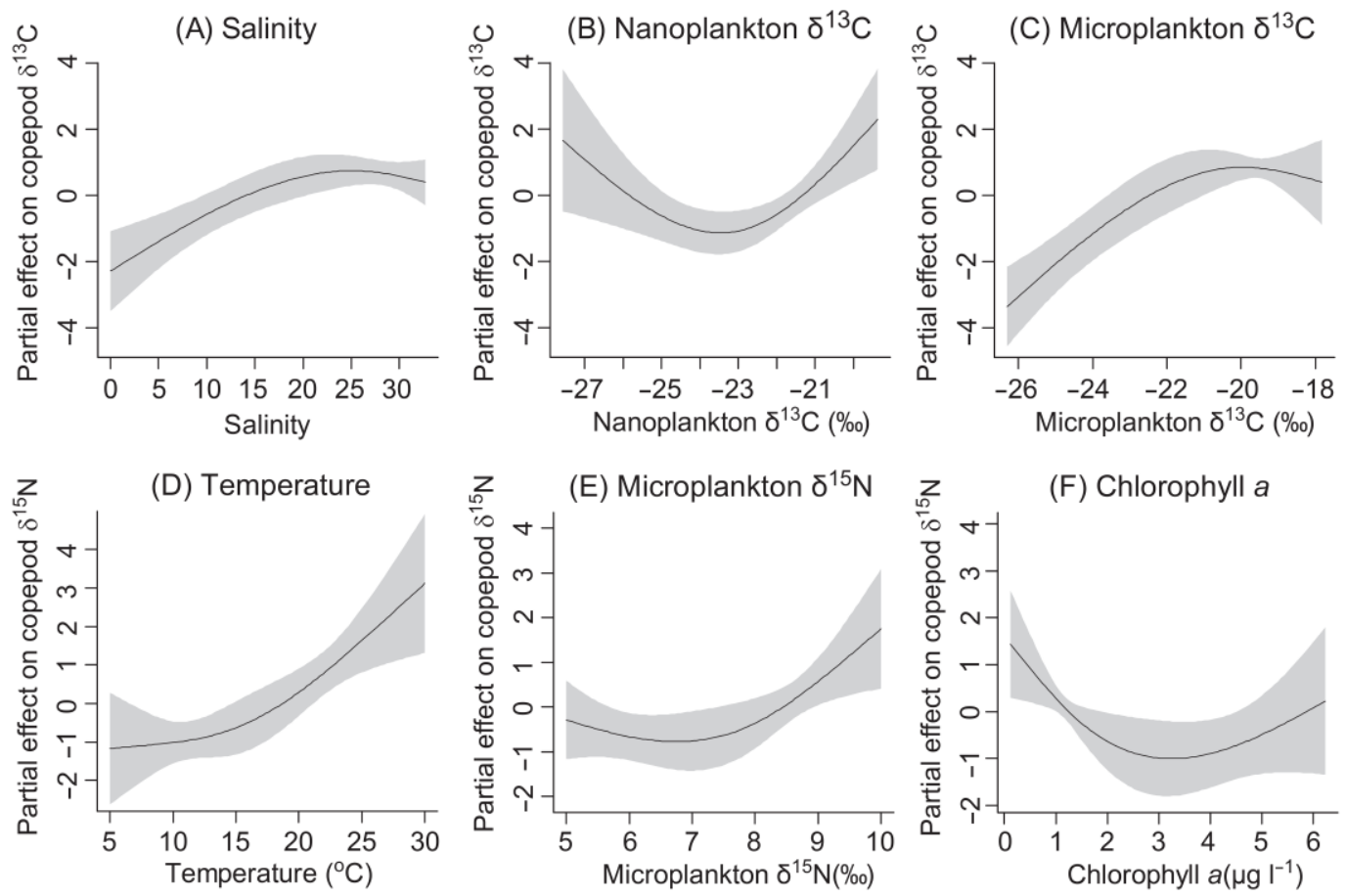

Figure S1: Partial effects of important environmental factors on the variabilities in stable isotopes. 

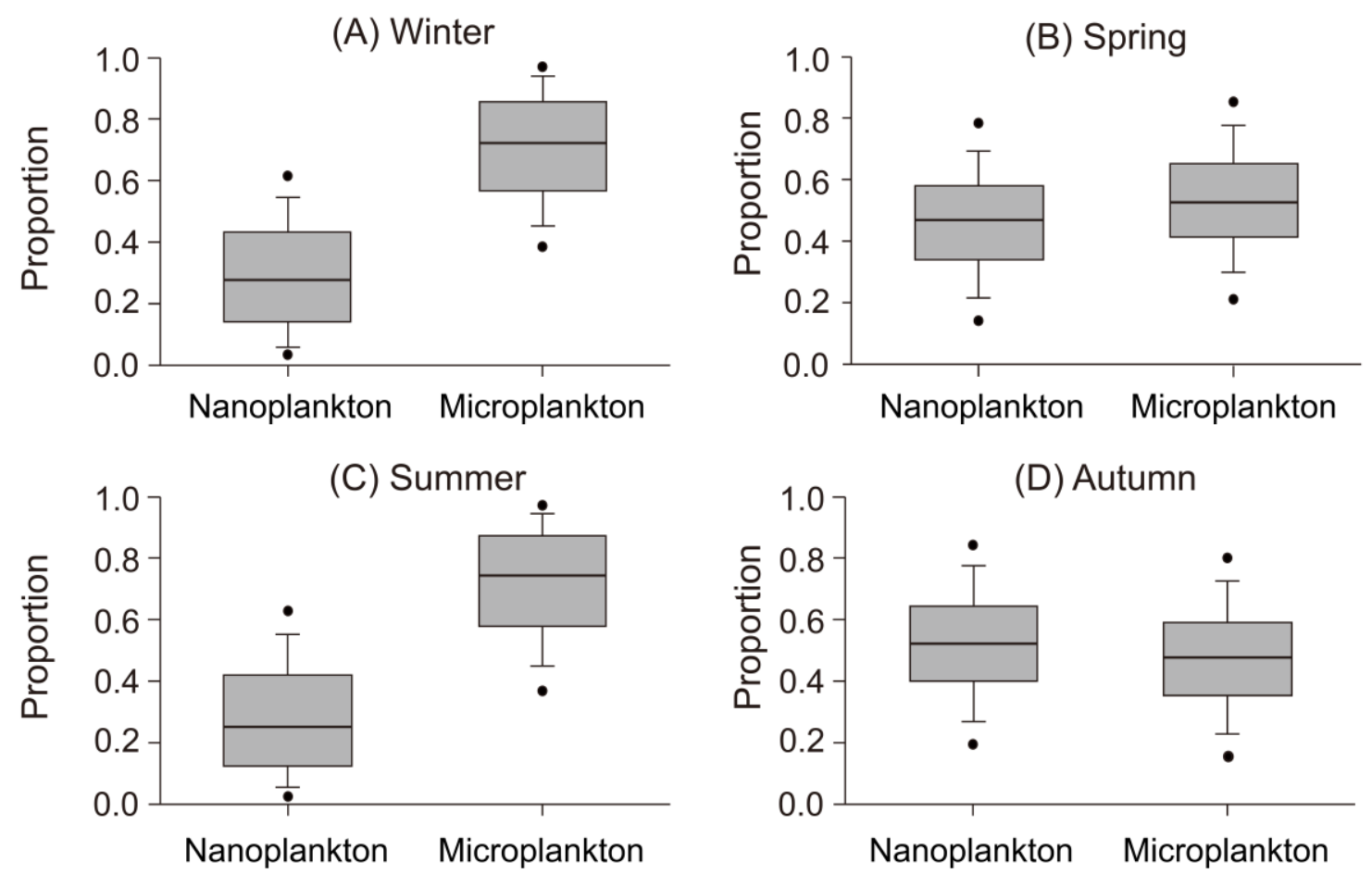

Figure S2: Temporal variations in the contributions of size-fractionated plankton in copepod diets estimated by a Bayesian mixing model using the SIAR package in the $R$ statistical program. Credibility intervals of 95\% (dots), $75 \%$ (whiskers), and $25 \%$ (boxes) and mean values (lines in the boxes) are shown in boxplots for each season. 


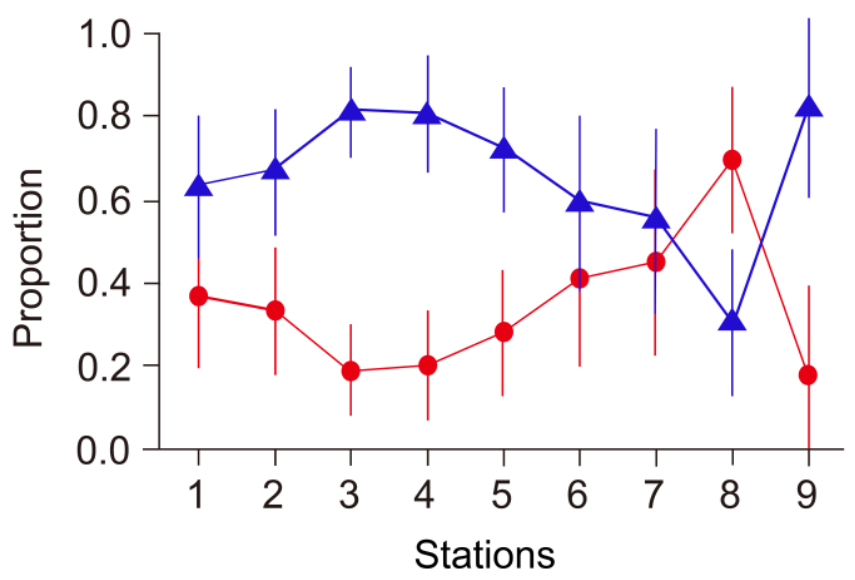

- Nanoplankton $\boldsymbol{\Delta}$ Microplankton

Figure S3: Spatial variations in the contributions of size-fractionated plankton in copepod diets estimated by Bayesian mixing model using the SIAR package. Mean values \pm standard deviations from all seasons are shown for each station. 